\title{
Comparative Study of Postoperative Complications in Patients With and Without an Obstruction Who Had Left- Sided Colorectal Cancer and Underwent a Single-Stage Operation After Mechanical Bowel Preparation
}

\author{
Sang Hun Jung, Jae Hwang Kim \\ Colorectal Division, Department of Surgery, Yeungnam University College of Medicine, Daegu, Korea
}

Purpose: The purpose of this study is to compare postoperative complications for single-stage surgery after mechanical bowel preparation in patients who experienced obstruction and those who did not.

Methods: From 2000 to 2011, 1,224 patients underwent a single-stage operation for left colorectal cancer after bowel preparation. Nonobstruction (NOB) and obstruction (OB) colorectal cancer patients were 1,053 (86.0\%) and 171 (14.0\%), respectively. Postoperative morbidity and mortality were compared between groups.

Results: The OB group had poor preoperative conditions (age, white blood cell, hemoglobin, albumin level, and advanced tumor stage) compared with the NOB group $(\mathrm{P}<0.05)$. Mean on-table lavage time for the OB group was 17.5 minutes (range, 14-60 minutes). Mean operation time for the OB group was statistically longer than that of the NOB group (OB: 210 minutes; range, $120-480$ minutes vs. NOB: 180 minutes; range, $60-420$ minutes; $\mathrm{P}<0.001)$. Overall morbidity was similar between groups (NOB: $19.7 \%$ vs. OB: $23.4 \%, \mathrm{P}=0.259$ ). Major morbidity was more common in the OB group than in the NOB group, but the difference was without significance (OB: $11.7 \%$ vs. NOB: $7.6 \%, \mathrm{P}=0.070$ ). Postoperative death occurred in 16 patients $(1.3 \%)$, and death in the OB group $(n=7)$ was significantly higher than it was in the NOB group $(n=9)(4.1 \%$ vs. $0.9 \%, P=0.001)$. Twelve patients had surgical complications, which were the leading cause of postoperative death: postoperative bleeding in five patients and leakage in seven patients.

Conclusion: Postoperative morbidity for a single-stage operation for obstructive left colorectal cancer is comparable to that for NOB, regardless of poor conditions of the patient.

Keywords: Single-stage operation; Obstruction; Left colorectal cancer; Morbidity; Mortality

\section{INTRODUCTION}

Approximately $15 \%-25 \%$ of colorectal patients present with obstruction $(\mathrm{OB})$ symptoms, and these usually require emergency

Received: September 4, 2014 • Accepted: October 29, 2014

Correspondence to: Jae Hwang Kim, M.D.

Department of Surgery, Yeungnam University College of Medicine,

170 Hyeonchung-ro, Nam-gu, Daegu 705-717, Korea

Tel: +82-53-620-3580, Fax: +82-53-624-1213

E-mail:98jshgs@hanmail.net

This was presented as a poster at ASCRS 2013, San Antonio, USA.

(c) 2014 The Korean Society of Coloproctology

This is an open-access article distributed under the terms of the Creative Commons Attribution NonCommercial License (http://creativecommons.org/licenses/by-nc/3.0) which permits unrestricted noncommercial use, distribution, and reproduction in any medium, provided the original work is properly cited. surgery. Emergency surgery for acute colonic $\mathrm{OB}$ is associated with a significant risk of mortality and morbidity [1]. The reason may be related to the fact that most of these patients are older and have a comorbidity, a fluid and electrolyte imbalance, and an advancedstage cancer [1]. As a result, the surgeon can select from a variety of surgical approaches, including a multistage operation, a Hartmann procedure, a single-stage operation, and a bridge to surgery using a metallic stent, that have been devised for the treatment of $\mathrm{OB}$ in patients with left-sided colorectal cancer [2].

Right-sided colonic OBs are usually treated by using a singlestage operation with primary anastomosis which has a complication rate than elective surgery; however, the optimal management of obstructive left colon cancer is still being debated. A multistage procedure has often been attempted because of the fear of anastomosis leakage and its related mortality [3]. However, this surgery 
has a high cumulative morbidity as a result of repeated major operations, and stoma-related morbidity is considerable [4]. Recently, a bridge to surgery using a metallic stent as a method to avoid emergency surgery or stoma formation has often been performed to treat patients with obstructed left colon cancer [5]. This procedure relieves the symptoms of $\mathrm{OB}$ symptom and improves the patient's condition, and compared with emergency surgery, an elective bridge to surgery for treating obstructive colon cancer reduces postoperative complications $[5,6]$. However, a perforation by the stent may induce fatal complications, such as peritonitis or sepsis, which can lead to mortality [7].

The single-stage operation is the standard surgery for treating patients with colorectal cancer because it is usually not associated with high postoperative morbidity and mortality. The benefit of bowel preparation in elective colon surgery has been questioned; however, surgery on an obstructed colon required a decompressed colon for the surgery to be feasible and anastomosis to be safe. Some authors have reported that a single-stage operation with colonic lavage or manual decompression has acceptable morbidity and mortality rates with a low leakage rate [8-11]; a colon cleaned by using intraoperative colonic irrigation may facilitate anastomosis healing, and in the event of leakage, patients with a clean colon appear to have fewer and less severe complication [12].

We consecutively performed single-stage surgery, which can be performed easily and safely by using a newly devised colonic irrigator, for the treatment of left-side colorectal-cancer patients presenting with OB after on-table lavage [13]. Reports on postoperative complications for patients with obstructive colorectal cancer treated with colonic lavage are few compared to those on nonobstruction (NOB) patients [8]. The purpose of this study is to identify postoperative complications in patients who underwent a single-stage operation after colonic lavage for treating obstructed leftsided colorectal cancer and to compare those complications with the ones observed in NOB patients.

\section{METHODS}

Between January 2000 and July 2011, we performed a restorative single-stage operation and intraoperative colonic irrigation for patients with obstructive colorectal cancer. All cases of left-sided colorectal cancer (from the splenic flexure to the rectum) during the same period were reviewed retrospectively by using data prospectively collected from the Colorectal Surgery Department. Patients having peritonitis, preoperative radiotherapy, nonresection of the primary lesion, no restorative surgery after resection, and a total colectomy were excluded from this study [13]. A total of 1,224 left-sided colorectal-cancer patients who underwent a single-stage operation were enrolled in this study.

Left colon $\mathrm{OB}$ was defined as an abdominal distention having gas passage disturbance and colon dilatation on radiologic findings such as those from clinical examination, abdominal radiography, and abdominopelvic computed tomography. Bowel preparation for colonic lavage was performed all patients before bowel anastomosis. In general, NOB patients underwent as standard mechanical bowel preparation using Colonyte with a 4 -L intake on the day before surgery; however, 48 patients underwent an intraoperative colonic irrigation in order to compare the efficacy of intraoperative lavage in patients without $\mathrm{OB}$ who underwent elective surgery to the efficacy of the standard preoperative mechanical bowel preparation. Bowel preparations of $\mathrm{OB}$ patients were performed using intraoperative colonic irrigation with a new intraoperative colonic irrigator (NICI, Dalim Co., Seoul, Korea) before the restorative procedure. Most patients with an $\mathrm{OB}$, after adequate fluid resuscitation, underwent surgery within 24 hours of admission. In all patients, prophylactic antibiotics with second-generation cephalosporin were administered before the initial incision.

Surgery was performed using either a laparotomy or a laparoscopic approach. Most cases of laparoscopic surgery at our center occurred in 2005 or later years. The procedure for single-stage surgery for treating patients with left-sided colorectal cancer is as follows: Patients were placed in the lithotomy position under general anesthesia. Before mobilization of the colon, the inferior mesentery artery was divided and ligated at the level of the low or high tie, depending on the surgeon's preference, and the inferior mesentery vein was ligated separately. Then, the left colon and the rectum, with or without splenic flexure division, were mobilized as much as necessary to allow a tension-free anastomosis with sufficient blood supply to the proximal colon. Depending on the tumor's location, distal resection of the sigmoid colon or rectum was done using a stapler. The anvil head was inserted via a proximal colostomy. The majority of anastomoses for OB were fashioned side to end by using a circular stapler to reduce the effects of size disparity. For most NOB patients, the anastomoses were fashioned end to end by using a circular stapler. Closed suction drainage was placed on the left paracolic gutter and pelvis. Depending on the surgeon's decision, either a loop ileostomies $(\mathrm{n}=$ 68 ) or a T-loop colostomy $(n=1)$ was performed to protect the anastomosis after single-stage surgery; 15 of 68 loop ileostomy was an OB group.

The detailed procedure for intraoperative colonic irrigation using NICI is as follows [13]: A 10-cm-long segment is decompressed 5 to $10 \mathrm{~cm}$ above the lesion. Intestinal clamps are then used to perform double clamping. While the sidewall of the decompressed colon is pulled using Allys forceps, a purse-string clamp is applied. This places a purse-string suture around the hole made by resection of a small segment of the colon wall. The y-shaped NICI connection is inserted into this hole, the purse-string suture is tightened, and the fastening nut is turned for fixation so that the colon will not be disconnected. To reinforce this connecting fixation, the fastening tie is looped around the connection and fastened. Afterward, when the intestinal clamps are released, the accumulated colonic contents gush out into the NICI and come out through the drainage tube, to be collected in the collecting bag on the floor. The operator can use his hands to squeeze the proximal colon gen- 
tly in the direction of drainage to help discharge fecal matter. Then, the irrigation catheter, which is connected to a large container that is less than $80 \mathrm{~cm}$ above the operating table, is inserted through the branch tube of the irrigator and made to reach the cecum. Saline is infused through the irrigation catheter, which dilutes the colon's contents to allow drainage. When the drained fluid is clear, the irrigation catheter is removed.

NOB and OB patients who underwent a single-stage operation after colonic lavage were compared in terms of postoperative morbidity and mortality. Clinicopathologic variables (sex, age, body mass index, TNM status), American Society of Anesthesiologists (ASA) score, and laboratory data were evaluated preoperatively in all patients. Postoperative morbidity included surgical site infection (wound infection and abscess), anastomosis leakage, postoperative bleeding, small bowel OB, pseudomembranous colitis, urinary tract infection, pneumonia, acute lung injury, sepsis after surgery, cardiovascular events, and acute renal failure. Major morbidity was defined as cases requiring a radiologic, endoscopic, or surgical procedure for management of the complication and resulting in organ failure (myocardial infarction, cerebrovascular infarction or hemorrhage, renal failure requiring dialysis, respiratory failure requiring ventilator care) [14]. All postoperative morbidities occurring in the same patient were included. Postoperative mortality was defined as death occurring within the first 30 days of the postoperative course or during the same hospitalization after the surgical procedure.

Continuous data are expressed as mean \pm standard deviation, and the range is given in parentheses. Continuous data were compared using Fisher exact test. Categorical data were compared using the chi-square test. Two-tailed $\mathrm{P}<0.05$ was considered statistically significant. The PASW Statistics ver. 18.0 (SPSS Inc., Chicago, IL, USA) was used for management of patient data and for statistical calculations.

\section{RESULTS}

\section{Patient characteristics}

Patient characteristics are detailed in Table 1. A total of 1,224 patients underwent a single-stage operation for left-sided colorectal cancer with colonic lavage before restorative procedures: 171 $(14.0 \%)$ in the OB group and 1,053 (86.0\%) in the NOB group. Fifteen patients in the NOB group, who initially presented with an $\mathrm{OB}$, were treated as bridge to surgery by using a self-expanding metallic stent. Forty-eight NOB patients were treated intraoperatively with colonic lavage, and the mean intraoperative irrigation time for the NOB group was 12.3 minutes (range: 5-20 minutes) compared with 17.5 minutes (range: $6-40$ minutes) in the $\mathrm{OB}$ group $(\mathrm{P}=0.001)$.

Age older than 70 years was significantly common in the $\mathrm{OB}$ group compared with the NOB group $(\mathrm{P}=0.043)$. The male-to-female ratios for the two groups were similar. The ASA scores were comparable between the two groups, and 62 emergency operations
Table 1. Clinicopathologic characteristics of obstruction (OB) and nonobstruction (NOB) left-sided colorectal cancer patients who underwent a single-stage operation

\begin{tabular}{|c|c|c|c|}
\hline Characteristic & NOB & $\mathrm{OB}$ & P-value \\
\hline No. of patients (total $=1,224$ ) & $1,053(86.0)$ & $171(14.0)$ & \\
\hline Sex & & & 0.821 \\
\hline Female & 403 (38.3) & 67 (39.2) & \\
\hline Male & $650(61.7)$ & $104(60.8)$ & \\
\hline Age (yr) & & & 0.043 \\
\hline$<70$ & $763(72.5)$ & $111(64.9)$ & \\
\hline$\geq 70$ & $290(27.5)$ & $60(35.1)$ & \\
\hline CEA (ng/mL) & & & $<0.010$ \\
\hline$<5$ & $748(71.0)$ & $97(56.7)$ & \\
\hline$\geq 5$ & $305(29.0)$ & $74(43.3)$ & \\
\hline ASA score & & & 0.248 \\
\hline $1 / 2$ & $991(94.1)$ & $157(91.8)$ & \\
\hline $3 / 4$ & $62(5.9)$ & $14(8.2)$ & \\
\hline 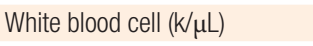 & & & $<0.010$ \\
\hline $4.0-10.0$ & $934(88.7)$ & $118(69.0)$ & \\
\hline$\leq 4.0$ or $\geq 10.0$ & $119(11.3)$ & $53(31.0)$ & \\
\hline Hemoglobin (g/dL) & & & $<0.010$ \\
\hline$\geq 10.0$ & $1,010(95.8)$ & $152(88.8)$ & \\
\hline$<10.0$ & $43(4.2)$ & $19(11.2)$ & \\
\hline Albumin (g/dL) & & & $<0.01$ \\
\hline$\geq 3.5$ & $1,010(95.8)$ & $113(66.1)$ & \\
\hline$<3.5$ & $43(4.2)$ & $58(33.9)$ & \\
\hline Body mass index (kg/m²) & & & $<0.010$ \\
\hline$\leq 27$ & $907(86.1)$ & $164(96.1)$ & \\
\hline$>27$ & $146(13.9)$ & $7(3.9)$ & \\
\hline Operation status & & & $<0.010$ \\
\hline Elective & $1,051(99.8)$ & $109(63.7)$ & \\
\hline Emergency & $2(0.2)$ & $62(36.3)$ & \\
\hline Surgical approach & & & $<0.01$ \\
\hline Open & $555(52.7)$ & $171(100.1)$ & \\
\hline Laparoscopy & 498 (47.3) & $0(0)$ & \\
\hline Operation time (min) & $180(60-420)$ & $210(120-480)$ & $<0.010$ \\
\hline On-table lavage time (min) & $12.3(4-20)$ & $17.5(14-60)$ & 0.001 \\
\hline Tumor location & & & 0.275 \\
\hline Left colon & $581(55.2)$ & $102(59.2)$ & \\
\hline Rectum & $472(44.8)$ & $69(40.4)$ & \\
\hline Protective stoma & & & 0.055 \\
\hline No & $999(94.9)$ & $156(91.2)$ & \\
\hline Yes & $54(5.1)$ & $15(8.8)$ & \\
\hline Depth of invasion & & & $<0.010$ \\
\hline $\mathrm{T} 1 / 2$ & $338(32.1)$ & $3(1.8)$ & \\
\hline T3 & $619(58.8)$ & $107(62.6)$ & \\
\hline T4 & $96(9.1)$ & $61(35.7)$ & \\
\hline Nodal status & & & $<0.010$ \\
\hline No & $619(58.8)$ & $64(37.4)$ & \\
\hline N1 & $285(27.1)$ & $50(29.2)$ & \\
\hline N2 & $149(14.2)$ & 57 (33.3) & \\
\hline Distant metastasis & & & $<0.010$ \\
\hline No & $944(89.6)$ & $125(73.1)$ & \\
\hline Yes & $109(10.4)$ & 46 (26.9) & \\
\hline LOHS (day) & $12.7(4-93)$ & $15.8(6-130)$ & $<0.010$ \\
\hline
\end{tabular}

Values are presented as number (\%) or median (range).

CEA, carcinoembryonic antigen; ASA, American Society of Anesthesiologists; LOHS, Length of postoperative hospital stay. 
Table 2. Methods of single-stage surgery for 1,224 left-sided cololectal cancer patients

\begin{tabular}{lcc}
\hline Operation method & NOB $(n=1,053)$ & OB $(n=171)$ \\
\hline Left hemicolectomy & $71(6.7)$ & $26(15.2)$ \\
Anterior resection & $487(46.2)$ & $67(39.2)$ \\
Low anterior resection & $405(38.5)$ & $70(40.9)$ \\
Ultralow anterior resection & $87(8.3)$ & $8(4.7)$ \\
Extended colectomy & $3(0.6)^{\mathrm{a}}$ & $2(1.2)^{\mathrm{b}}$ \\
\hline
\end{tabular}

Values are presented as number (\%).

NOB, nonobstruction; OB, obstruction

${ }^{a}$ Extended transverse colon resection due to a synchronous lesion and ${ }^{b}$ left colon necrosis after inferior mesentery artery ligation.

(36.3\%) were performed in the OB group. Preoperative laboratory data, including white blood cell (WBC), hemoglobin, and albumin, were abnormal in the OB group compared with the NOB group (P $<0.001)$. The mean operation time was longer in the $\mathrm{OB}$ group than in the NOB group (180 minutes vs. 210 minutes, $\mathrm{P}<0.001$ ). Tumor pathologic features of the OB group were significantly more advanced than those of the NOB group (preoperative carcinoembryonic antigen level TNM status). The postoperative mean hospital stay was 15.8 days (range, $6-136$ days) in the OB group compared with 12.7 days (range, $4-93$ days) in the NOB group $(\mathrm{P}<0.001)$.

A standard segmental resection was performed in most patients (Table 2): 1,047 of 1,053 (99.4\%) in the NOB group and 169 of 171 $(98.8 \%)$ in the OB group (Table 2). Two patients in the OB group were initially treated using a standard resection; however, an extended colectomy was finally performed due to left colon necrosis after inferior mesentery artery (IMA) ligation. Three NOB patients were also treated using an extended colectomy due to synchronous proximal cancer. Sixty-nine patients received a protective stoma (Table 3). Common reasons for protective-stoma construction in $\mathrm{OB}$ patients were anastomosis safety in 5 patients, followed by 4 patients with a $3 / 4$ ASA score. All protective stomas in $\mathrm{OB}$ patients were closed.

\section{Postoperative complications}

All postoperative complications observed in both groups are summarized in Table 4. Of 1,224 patients, 247 patients (20.2\%) had postoperative complications, and no significant differences were observed between the groups (NOB, $19.7 \%$ vs. OB, $23.4 \%$; $=$ 0.259 ). Small bowel $\mathrm{OB}$ was the most common complication, 72 patients (6.6\%) in the NOB group and 12 patients (7.0\%) in the $\mathrm{OB}$ group, and its rate of occurrence was similar between the two groups $(\mathrm{P}=0.964)$. Clinical anastomotic leakage developed in 63 patients (5.1\%), and the incidence of leakage was similar between the two groups: 54 patients (5.1\%) in the NOB group and 9 patients $(5.3 \%)$ in the $\mathrm{OB}$ group $(\mathrm{P}=0.941)$. Three of the nine protective fecal-diversion patients in the $\mathrm{OB}$ group showed minor leakage symptoms, and the stomas of all nine patients were closed within six months postoperatively. The rate of wound infection in the $\mathrm{OB}$
Table 3. Details of 69 protective fecal diversion cases

\begin{tabular}{lcc}
\hline Variable & NOB $(\mathrm{n}=54)$ & $\mathrm{OB}(\mathrm{n}=15)$ \\
\hline Type of operation & 54 & 14 \\
Loop ileostomy & 0 & 1 \\
Transverse loop colostomy & 3 & 7 \\
$\begin{array}{l}\text { Tumor location } \\
\text { Colon }\end{array}$ & 51 & 8 \\
Rectum & & \\
Main cause of protective stoma & & \\
construction & 4 & 4 \\
ASA 3/4 & 4 & 5 \\
Problem of anastomosis safety & 6 & 3 \\
Difficult procedure & $12 / 28$ & $1 / 2$ \\
Pouch procedure/lower level of & & \\
$\quad$ anastomosis & & 3 \\
Postoperative results & 9 & 0 \\
Leakage & 1 (myocardial infarction) \\
Mortality & $5^{\mathrm{a}}$ & \\
Cause of failure of stoma reduction &
\end{tabular}

NOB, nonobstruction; OB, obstruction; ASA, American Society of Anesthesiologists. ${ }^{a}$ Cause as permanent stoma: death (1), rectovaginal fistula (3), conversion to end colostomy due to colon necrosis (1).

group (3.5\%) was comparable to that in the NOB group (2.1\%) (P $=0.249$ ). Intra-abdominal abscess developed in seven patients $(0.6 \%)$, and its rates of occurrence were similar in both groups. However, wound evisceration occurred more frequently in the $\mathrm{OB}$ group $(2.3 \%)$ than in the NOB group $(0.8 \%)$, and this difference showed marginal significance $(\mathrm{P}=0.052)$.

Major postoperative morbidity occurred in 100 patients (8.2\%). Major morbidity in the $\mathrm{OB}$ group was more common than it was in the NOB group: however, the difference was not significant (OB, $11.7 \%$ vs. NOB, $7.6 \% ; \mathrm{P}=0.070$ ). The causes of major morbidity were as follows: 41 patients with anastomotic leakage, six patients, five patients, two patients, one patients with ileostomy prolapse, 1 patient with a cerebrovascular attack, and 1 patient with an acute lung injury.

\section{Postoperative mortality}

Deaths that occurred until postoperative day 30 are summarized in Table 5. Postoperative death occurred in 16 patients (1.3\%), and death in the OB group $(\mathrm{n}=7)$ was significantly higher than in the NOB group $(n=9)(4.1 \%$ vs. $0.9 \%, P=0.001)$. Twelve patients had surgical complication as the leading cause of postoperative death: postoperative bleeding and subsequent renal failure in five patients and leakage in seven patients. Three patients had medical causes of death: myocardial infarction in two patients, and acute renal failure in one patient who had not undergone hemodialysis. One patient died due to cancer progression with multiple metas- 
Table 4. Postoperative complications for obstruction (OB) and nonobstruction (NOB) left-sided colorectal cancer patients who underwent a single-stage operation

\begin{tabular}{|c|c|c|c|c|}
\hline Postoperative complication & Total $(n=1,224)$ & NOB $(n=1,053)$ & $\mathrm{OB}(\mathrm{n}=171)$ & P-value \\
\hline All morbidity & $247(20.2)$ & $207(19.7)$ & $40(23.4)$ & 0.259 \\
\hline Anastomoses leakage & $63(5.1)$ & $54(5.1)$ & $9(5.3)$ & 0.941 \\
\hline Intraabdominal abscess & $7(0.6)$ & $5(0.5)$ & $2(1.2)$ & 0.264 \\
\hline Wound dehiscence & $12(1.0)$ & $8(0.8)$ & $4(2.3)$ & 0.052 \\
\hline Postoperative bleeding & $21(1.6)$ & $17(2.3)$ & $4(1.7)$ & 0.498 \\
\hline Acute renal failure & $23(1.8)$ & $19(1.9)$ & $4(2.3)$ & 0.633 \\
\hline Pseudomembranous colitis & $12(1.0)$ & $12(1.1)$ & $0(0)$ & \\
\hline Urinary tract infection & $4(0.3)$ & $3(0.3)$ & $1(0.6)$ & \\
\hline Upper gastrointestinal bleeding & $3(0.2)$ & $2(0.2)$ & $1(0.6)$ & \\
\hline Neurogenic bladder & $2(0.2)$ & $2(0.2)$ & $0(0)$ & \\
\hline Pneumonia or acute lung injury & $4(0.3)$ & $2(0.2)$ & $2(1.2)$ & \\
\hline Unknown sepsis & $2(0.2)$ & $2(0.2)$ & $0(0)$ & \\
\hline Stoma complication & $4(0.3)$ & $4(0.4)$ & $0(0)$ & \\
\hline Major morbidity & $100(8.2)$ & $80(7.6)$ & $20(11.7)$ & 0.070 \\
\hline Anastomosis leakage & $41(4.0)$ & $35(3.5)$ & $6(3.5)$ & \\
\hline Intra-abdominal abscess & $6(0.5)$ & $5(0.5)$ & $1(0.6)$ & \\
\hline Wound eviceration & $10(0.9)$ & $7(0.8)$ & $3(1.8)$ & \\
\hline Cerebrovascular attack & $1(0.1)$ & $1(0.1)$ & $0(0)$ & \\
\hline Acute lung injury & $1(0.1)$ & $1(0.1)$ & $0(0)$ & \\
\hline
\end{tabular}

Values are presented as number (\%).

tases. All patients who died due to anastomotic leakage did not have a protective stoma.

\section{DISCUSSION}

Although a number of studies on left colon emergencies have been published, reports on postoperative complications in obstructedcolon patients compared with non-obstructed-colon patients are extremely rare [8]. Primary resection with an anastomosis offers the advantage of a definite procedure without the need for further surgery. However, the limitations of this procedure are the techni- cal challenge and the potential risk of a high postoperative complication rate, depending on the general preoperative conditions or fecal-loading status of the colon [15]. The present study revealed that postoperative morbidity for left-colon $\mathrm{OB}$ patients who underwent a single-stage operation after on-table lavage did not significantly differ from that for NOB patients, despite the poor preoperative condition of the patients [16]. However, postoperative death in $\mathrm{OB}$ patients was statistically higher than it was in NOB patients and was closely related to postoperative major surgical complications.

Most of colon-cancer $\mathrm{OB}$ patients have an adverse preoperative 
Table 5. Details of the 16 postoperative mortality cases

\begin{tabular}{|c|c|c|c|c|c|c|c|c|}
\hline No. & Age (yr) & Sex & Obstruction & Stoma & ASA & Postoperative main events & Treatment & LOHS \\
\hline 1 & 79 & $M$ & Yes & No & $3 \mathrm{E}$ & Bleeding, ARF & Cons & 26 \\
\hline 2 & 64 & M & Yes & No & $3 \mathrm{E}$ & Leakage, sepsis & Cons & 22 \\
\hline 3 & 82 & M & Yes & No & $2 \mathrm{E}$ & Bleeding, ARF, sepsis & Cons & 9 \\
\hline 4 & 64 & M & Yes & No & $3 \mathrm{E}$ & SBO, small bowel leakage, ARF & Surg & 27 \\
\hline 5 & 69 & M & Yes & No & $3 \mathrm{E}$ & Cancer progress & Cons & 25 \\
\hline 6 & 62 & M & Yes & No & $3 \mathrm{E}$ & Bleeding, ARF & Cons & 9 \\
\hline 7 & 74 & $\mathrm{~F}$ & Yes & No & $2 \mathrm{E}$ & Leakage (ischemia), sepsis & Surg & 13 \\
\hline 8 & 73 & M & No & No & 2 & Leakage (ischemia), sepsis & Surg & 15 \\
\hline 9 & 81 & M & No & No & 1 & Leakage, sepsis & Surg & 21 \\
\hline 10 & 80 & M & No & Yes & 3 & Myocardial infaction & Cons & 17 \\
\hline 11 & 68 & $\mathrm{~F}$ & No & No & 2 & Anastomosis bleeding, ARF & Surg & 7 \\
\hline 12 & 74 & M & No & No & 2 & ARF, leakage, sepsis & Cons & 29 \\
\hline 13 & 57 & M & No & No & 2 & Bleeding, ARF, acute lung injury & Cons & 4 \\
\hline 14 & 86 & $\mathrm{~F}$ & No & No & 2 & ARF, sepsis & Cons & 23 \\
\hline 15 & 75 & M & No & No & 1 & SBO, Myocardial infaction & Surg & 26 \\
\hline 16 & 81 & M & No & No & 2 & Leakage, SB perforation, ARF & Surg & 21 \\
\hline
\end{tabular}

ASA, american society of anesthesiologists; LOHS, length of hospital stay; ARF, acute renal failure; Cons, conservative treatment; SBO, small bowel obstruction; Surg, surgical treatment; SB, small bowel obstruction.

Details of surgery: №. 4, adhesiolysis; 7, 8, 9, Hartmann operation; 11, reanastomosis; 15, adhesiolysis; 16, ileostomy.

condition $[17,18]$. The present study also revealed that the preoperative status in $\mathrm{OB}$ patients was worse than it was in $\mathrm{NOB}$ patients in terms of older age, abnormal WBC count, and lower serum albumin and hemoglobin levels. Some preoperative laboratory findings are partially corrected before emergency surgery for $\mathrm{OB}$ patients; however, not all these factors are closely associated with postoperative complications [17]. Colorectal cancer $\mathrm{OB}$ is frequent in elderly patients with a variety of medical diseases, so age is generally considered to be an important risk factor for postoperative morbidity and mortality [16]. The cardiopulmonary function is also aggravated by abdominal distention, which develops after a large bowel OB; however this may be relieved after surgery. In this study, the postoperative morbidity for OB patients was not significantly higher than that for NOB patients, regardless of the poor preoperative condition of the $\mathrm{OB}$ patients.

The dilemma of surgery for left-sided colon $\mathrm{OB}$ is surgical safety for the dilated colon and the fecal-loaded colon. A variety of procedures have been applied for left-sided colon OB; however, a single-stage operation has usually been performed for right-colon $\mathrm{OB}$, with high postoperative morbidity. A multistaged operation for obstructive left-colon cancer involves repeated major surgery with significant cumulative morbidity and mortality, significant costs, and a significant stoma-related problem with $50 \%$ permanent status. Bridge to surgery using a stent, when it was successful, showed a reduction in postoperative morbidity and mortality [6]. However, the safety of the stent procedure has not yet been confirmed, with failure of a randomized trial [7, 18]. A single-stage operation for obstructive left-colon cancer has usually been reported as an on-table lavage or manual decompression, depending on the surgeon's preference and technical ease [11, 12]. Manual decompression of the fecal load has a shorter operation time than on-table lavage; however, incomplete bowel preparation load may induce anastomosis leakage, which is a fatal complication [9, $19,20]$. The purpose of irrigation is to achieve a colon free of feces, decreasing the rate of suture failure and its attendant complications [21]. Some concerns regarding on-table lavage include an extension of the operation time and the need for a surgeon experienced in the procedure $[13,22]$. The time required for on-table lavage ranges from 20 to 60 minutes, but this procedure is really not long and risky compared with manual decompression or bridge to surgery using a stent [8]. In addition, on-table lavage may be fully acceptable if surgeons do not consider it a troublesome task $[9,16,23]$. In this study, 11 years of consecutive results for a colorectal fellowship-trained surgeon support the conclusion that a single-stage operation after on-table lavage by using a welldesigned device, compared with NOB surgery, is an acceptable procedure that has a procedure time of approximately 25 minutes and avoids contamination [8].

In this study, approximately $23 \%$ of the OB patients who underwent a single-stage operation had postoperative complications, which was comparable to that of NOB patients. This result was consistent with other reports on a single-stage operation (23\% to 
25.3\%) $[9,16]$. Interestingly, contrary to other results, cardiopulmonary complications developed after OB surgery in a small number of patients [23,24]. The most common complication was small bowel $\mathrm{OB}$, followed by anastomosis leakage and wound infection [24]. These complications were similar tgo those for the NOB patients. The incidence of wound infection after a single-stage operation using NICI was 3.5\%, which was lower than other reported values, which ranged from $5.4 \%$ to $11 \%$ [21, 24. 25]. In addition, the rate of intraabdominal abscess was low, $1.2 \%$, after surgery. We observed considerable major morbidity in $\mathrm{OB}$ patients, but this finding was without statistical significance when compared to the value for NOB patients. Anastomotic leakage and postoperative acute renal failure following postoperative bleeding were common causes of major morbidity and were closely related with postoperative mortality [26].

The clinical anastomotic leakage rate for a single-stage operation after on-table lavage was $5.3 \%$ in this series and was comparable with that of NOB patients [26]. Recent reports have suggested that mechanical bowel preparation is not necessary for elective colon cancer surgery [27]. Some authors have reported that manual decompression to reduce fecal material before anastomosis in an obstructed colon does not significantly increase anastomotic leakage $[4,11]$. However, fecal loading and distended colon associated with $\mathrm{OB}$ may impair anastomosis collagen metabolism and healing, as shown in an experimental study [20,28]. Most results for a single-stage operation after colonic lavage in an obstructed colon showed leakage rates ranging from 0 to $6 \%[10,12,13,29]$. Sepsis following leakage is a fatal complication after colorectal surgery, and having a colon with less fecal loading may, at least, decrease septic complication following leakage [26]. In the present study, depending on the surgeon's decision, a simultaneous fecal diversion with a loop ileostomy, including a one-transverse-loop colostomy, was performed in $8.8 \%$ of OB patients, and no septic symptoms occurred in patients having a protective stoma, with all stoma having been closed within six months postoperatively.

In the present study, the postoperative death rate in $\mathrm{OB}$ patients was $4.1 \%$, which was not significantly different from the rate for NOB patients, and this value was slightly lower than the values published in other reports, which showed values of approximately $5 \%$ to $15 \%[9,16,30]$. Postoperative death in our series was commonly related with major surgical complications, such as postoperative bleeding or anastomotic leakage, although others researchers have reported frequent cardiopulmonary complications as a cause of death $[9,16]$. OB patients of older age and with comorbidity and poor preoperative condition might be highly vulnerable to a postoperative surgical complication, as in this result [17, 26]. Postoperative surgical complications such as bleeding may be prevented by using a secure procedure. In addition, patients with high risk for anastomotic leakage might be better managed with a protective fecal diversion, such as a loop ileostomy, which may decrease the need for additional surgery, as well as the leakage- related mortality [26].
This study included quite a large number of patients who underwent a single-stage operation with on-table lavage. Although the data were reviewed retrospectively, the results demonstrate that a single-stage operation with on-table lavage can safely be performed in the majority of patients with obstructed left-sided colorectal cancer, with the morbidity being similar to that for NOB patients.

\section{CONFLICT OF INTEREST}

No potential conflict of interest relevant to this article was reported.

\section{ACKNOWLEDGMENTS}

This work was supported by the 2013 Yeungnam University Research Grant.

\section{REFERENCES}

1. Gainant A. Emergency management of acute colonic cancer obstruction. J Visc Surg 2012;149:e3-10.

2. Ansaloni L, Andersson RE, Bazzoli F, Catena F, Cennamo V, Di Saverio $\mathrm{S}$, et al. Guidelenines in the management of obstructing cancer of the left colon: consensus conference of the world society of emergency surgery (WSES) and peritoneum and surgery (PnS) society. World J Emerg Surg 2010;5:29.

3. Tan KK, Sim R. Surgery for obstructed colorectal malignancy in an Asian population: predictors of morbidity and comparison between left- and right-sided cancers. J Gastrointest Surg 2010;14: 295-302.

4. Villar JM, Martinez AP, Villegas MT, Muffak K, Mansilla A, Garrote $\mathrm{D}$, et al. Surgical options for malignant left-sided colonic obstruction. Surg Today 2005;35:275-81.

5. Driest JJ, Zwaving HH, Ledeboer M, Eeftinck Schattenkerk M, Kuipers EJ, Ter Borg F. Low morbidity and mortality after stenting for malignant bowel obstruction. Dig Surg 2011;28:367-71.

6. Cennamo V, Luigiano C, Manes G, Zagari RM, Ansaloni L, Fabbri $\mathrm{C}$, et al. Colorectal stenting as a bridge to surgery reduces morbidity and mortality in left-sided malignant obstruction: a predictive risk score-based comparative study. Dig Liver Dis 2012;44: 508-14.

7. Tan CJ, Dasari BV, Gardiner K. Systematic review and meta-analysis of randomized clinical trials of self-expanding metallic stents as a bridge to surgery versus emergency surgery for malignant left-sided large bowel obstruction. Br J Surg 2012;99:469-76.

8. Sasaki K, Kazama S, Sunami E, Tsuno NH, Nozawa H, Nagawa H, et al. One-stage segmental colectomy and primary anastomosis after intraoperative colonic irrigation and total colonoscopy for patients with obstruction due to left-sided colorectal cancer. Dis Colon Rectum 2012;55:72-8.

9. Baccari P, Bisagni P, Crippa S, Sampietro R, Staudacher C. Operative and long-term results after one-stage surgery for obstructing 
colonic cancer. Hepatogastroenterology 2006;53:698-701.

10. Single-stage treatment for malignant left-sided colonic obstruction: a prospective randomized clinical trial comparing subtotal colectomy with segmental resection following intraoperative irrigation. The SCOTIA Study Group. Subtotal Colectomy versus On-table Irrigation and Anastomosis. Br J Surg 1995;82:1622-7.

11. Lim JF, Tang CL, Seow-Choen F, Heah SM. Prospective, randomized trial comparing intraoperative colonic irrigation with manual decompression only for obstructed left-sided colorectal cancer. Dis Colon Rectum 2005;48:205-9.

12. Kam MH, Tang CL, Chan E, Lim JF, Eu KW. Systematic review of intraoperative colonic irrigation vs. manual decompression in obstructed left-sided colorectal emergencies. Int J Colorectal Dis 2009;24:1031-7.

13. Kim JH, Shon DH, Kang SH, Jang BI, Chung MK, Kim JH, et al. Complete single-stage management of left colon cancer obstruction with a new device. Surg Endosc 2005;19:1381-7.

14. Dindo D, Demartines N, Clavien PA. Classification of surgical complications: a new proposal with evaluation in a cohort of 6336 patients and results of a survey. Ann Surg 2004;240:205-13.

15. Grundmann RT. Primary colon resection or Hartmann's procedure in malignant left-sided large bowel obstruction? The use of stents as a bridge to surgery. World J Gastrointest Surg 2013;5:1-4.

16. Chiappa A, Zbar A, Biella F, Staudacher C. One-stage resection and primary anastomosis following acute obstruction of the left colon for cancer. Am Surg 2000;66:619-22.

17. McGillicuddy EA, Schuster KM, Davis KA, Longo WE. Factors predicting morbidity and mortality in emergency colorectal procedures in elderly patients. Arch Surg 2009;144:1157-62.

18. Alcantara M, Serra-Aracil X, Falco J, Mora L, Bombardo J, Navarro $\mathrm{S}$. Prospective, controlled, randomized study of intraoperative colonic lavage versus stent placement in obstructive left-sided colonic cancer. World J Surg 2011;35:1904-10.

19. Schrock TR, Deveney CW, Dunphy JE. Factor contributing to leakage of colonic anastomoses. Ann Surg 1973;177:513-8.

20. Irvin TT. Collagen metabolism in infected colonic anastomoses. Surg Gynecol Obstet 1976;143:220-4.
21. Ortiz H, Biondo S, Ciga MA, Kreisler E, Oteiza F, Fraccalvieri D. Comparative study to determine the need for intraoperative colonic irrigation for primary anastomosis in left-sided colonic emergencies. Colorectal Dis 2009;11:648-52.

22. Konishi F, Muto T, Kanazawa K, Morioka Y. Intraoperative irrigation and primary resection for obstructing lesions of the left $\mathrm{co}^{-}$ lon. Int J Colorectal Dis 1988;3:204-6.

23. Murray JJ, Schoetz DJ Jr, Coller JA, Roberts PL, Veidenheimer MC. Intraoperative colonic lavage and primary anastomosis in nonelective colon resection. Dis Colon Rectum 1991;34:527-31.

24. Hsu TC. Comparison of one-stage resection and anastomosis of acute complete obstruction of left and right colon. Am J Surg 2005; 189:384-7.

25. Biondo S, Pares D, Frago R, Marti-Rague J, Kreisler E, De Oca J, et al. Large bowel obstruction: predictive factors for postoperative mortality. Dis Colon Rectum 2004;47:1889-97.

26. Bakker IS, Grossmann I, Henneman D, Havenga K, Wiggers T. Risk factors for anastomotic leakage and leak-related mortality after colonic cancer surgery in a nationwide audit. Br J Surg 2014;101: 424-32.

27. Slim K, Vicaut E, Launay-Savary MV, Contant C, Chipponi J. Updated systematic review and meta-analysis of randomized clinical trials on the role of mechanical bowel preparation before colorectal surgery. Ann Surg 2009;249:203-9.

28. Duraker N, Bender O, Memisoglu K, Yalciner A. Intraoperative bowel irrigation improves anastomotic collagen metabolism in the left-sided colonic obstruction but not covering colostomy. Int J Colorectal Dis 1998;13:232-4.

29. Park UC, Chung SS, Kim KR, Seong MK, Yoon WH, Kim YJ, et al. Single-stage procedure with intraoperative colonoscopy and colonic irrigation in patients with obstructing left-sided colonic cancer. Int J Colorectal Dis 2004;19:487-92.

30. Sim WJ, Park YK, Chi KC, Lee JH, Chang IT, Kim SJ. Comparison of clinical differences between colonic obstruction and non-obstruction groups in colon surgery. J Korean Soc Coloproctol 2002; 18:65-72. 